\title{
Relación entre características de tipo y producción de leche en vacas Holstein de Antioquia, Colombia
}

\section{Relationship between type traits and milk production in Holstein cows from Antioquia, Colombia}

\author{
Juan Corrales A, ${ }^{1,2}$ Zoot, Mario Cerón-Muñoz, ${ }^{1}$ Ph.D, Jhon Cañas A, ${ }^{1}$ Zoot, \\ Cristina Herrera $\mathrm{R},{ }^{1}$ Zoot, Samir Calvo $\mathrm{C}^{1}{ }^{1}$ Zoot.
}

\begin{abstract}
${ }^{1}$ Universidad de Antioquia, Facultad de Ciencias Agrarias. Grupo de Investigación en Genética, Mejoramiento y Modelación Animal. ${ }^{2}$ Joven investigador Colciencias, programa jóvenes investigadores e innovadores "Virginia Gutiérrez de Pineda". *Correspondencia: mceronm@agronica.udea.edu.co.
\end{abstract}

Recibido: Mayo de 2010; Aceptado: Febrero de 2011.

\section{RESUMEN}

Objetivo. Evaluar la relación entre las características de tipo agrupadas por factores con la producción de leche en ganado Holstein de Antioquia, Colombia. Materiales y métodos. Se utilizaron datos de 24 características lineales y producción de leche de 3102 vacas de la raza Holstein del departamento de Antioquia en control lechero oficial. Se realizó un análisis por factores (AF) con el método de componentes principales y se retuvieron los factores que mostraron valores propios mayores que 1.0. Posteriormente se realizó un análisis de varianza para la variable producción de leche, donde se tuvieron en cuenta los efectos fijos de finca, mes de parto y año de clasificación y se estimaron los coeficientes de regresión lineal para cada uno de los factores retenidos. Resultados. El AF mostró que sólo siete factores fueron retenidos y agruparon cerca del 64\% del total de la varianza de todas las características de tipo analizadas. El primer factor reunió las variables relacionadas con la estructura general de la vaca y tuvo un valor propio de 3.85. El análisis de varianza mostró que los factores se relacionaron con producción de leche Conclusiones. Para producción de leche en Antioquia, Colombia, sobresalen las vacas grandes, anchas de pecho, altas y profundas del cuerpo, con pezuñas uniformes, angulosas y talones profundos, un sistema mamario caracterizado por ubres de textura suave, buen ligamento medio, un buen carácter lechero y ubres profundas.

Palabras clave: Análisis de componentes, control lechero, modelos lineales, producción lechera. (Fuentes: AIMS, CAB). 


\section{ABSTRACT}

Objective. To evaluate the relationship between type traits and milk production in Holstein cows from Antioquia, Colombia. Materials and methods. A total of 24 type traits and milk production from 3102 cows under an official milk control records were used. Data were analyzed by factors (FA) using the principal components method. Factors that showed eigenvalue greater than 1.0 were retained, and analyzed by ANOVA for production of milk, which took into account the fixed effects of farm, months of calving and year of evaluation. Then, linear regression coefficients were estimated for each of the factors retained. Results. The FA showed that only seven factors were retained, explaining about $64 \%$ of the total variance of all type traits analyzed. The first factor met the variables related to the overall structure of the cow and had an eigenvalue of 3.85. It was also found, that the factors studied were related mainly to milk production. Conclusions. The high milk production of dairy cows from Antioquia, Colombia, was associated to large cows, characterized by wide chest, tall and deep body, uniform feet and angular and deep heel and a mammary system characterized by soft texture, strong median suspensory ligament, deep udder and good dairy character.

Key words: Component analysis, linear models, milk production, milk recording. (Sources: AIMS, CAB).

\section{INTRODUCCIÓN}

Los rasgos morfológicos son la base de los sistemas de clasificación lineal en diferentes razas bovinas (1). Este sistema está basado en medidas visuales de características individuales desde un extremo biológico observable a otro el cual varía de 1 a 50 en el sistema de evaluación estadounidense y de 1 a 9 en los sistemas canadiense y europeo $(2,3)$.

La importancia de estas características está dada con la posibilidad de estar genotípica y fenotípicamente relacionadas con la longevidad y la habilidad para producción de leche (3).

Estudios tendientes a evaluar la relación entre las características de tipo con vida productiva y producción de leche, han sido comúnmente analizados por regresión lineal múltiple (4). Sin embargo, las altas correlaciones entre dichas características producen dificultad en su interpretación por efecto de la alta colinealidad, y por ende imprecisiones entre los estimativos de regresión (5).

Un enfoque estadístico para evitar dependencia entre las variables y desarrollar estimativos más ajustados, es el análisis factorial. Este procedimiento remueve la información redundante de variables correlacionadas, presentándolas en un pequeño grupo de variables derivadas llamadas factores (6) y permite agrupar variables que estén fuertemente relacionadas (7). De esta manera, los diferentes factores resultantes pueden ser utilizados en análisis posteriores, sin tener problemas de colinealidad.

El objetivo de este estudio fue evaluar la relación entre las características de tipo con la producción de leche en ganado Holstein de Antioquia.

\section{MATERIALES Y MÉTODOS}

Obtención de datos. Se utilizaron 3102 datos de producción de leche y clasificación lineal (sistema de 1 a 9) de vacas de primer parto, registradas en la Asociación Holstein de Colombia. Se contó con información de producción de leche ajustada a los 305 días y edad adulta (8), y evaluación lineal de 24 características de tipo: estatura, tamaño, ancho de pecho, profundidad del cuerpo, colocación de isquiones, ancho del isquion, calidad de hueso, colocación de los miembros, profundidad de la ubre, medio suspensorio, inserción anterior, colocación del pezón, largo del pezón y angularidad, basado en los parámetros utilizados por la Asociación Holstein de Colombia. Los datos fueron colectados entre los años 2000 y 2008. 
Análisis estadístico. Se utilizó la técnica multivariada de análisis por factores (AF) a través del procedimiento FACTOR del software estadístico $S A S \& 9.1$, con el fin de reducir la dimensionalidad y sintetizar la información contenida en un conjunto de $p$ variables observadas $(y 1, \ldots . ., y p)$ por la extracción de un nuevo conjunto de $\mathrm{m}$ factores subyacentes $(m<p)$ denotados por $f 1, f 2, \ldots . . ., f m$. El modelo empleado fue el siguiente:

$$
\begin{gathered}
X_{j}=\lambda_{j 1} f_{1}+\lambda_{2} f_{2}+\ldots . .+\lambda_{j m} f_{m}+\eta_{j} \\
\text { para } j=1,2, \ldots, p
\end{gathered}
$$

$X$ es el vector con las nuevas variables $f 1$, $f 2, \ldots, f_{m}$ son los factores comunes y $\eta_{1}, \eta_{2}$, ...., $\eta_{\mathrm{p}}$ factores específicos. La cantidad $\eta_{j}$ describe la variación residual específica a la j-ésima variable respuesta.

Los multiplicadores $\lambda_{\mathrm{jk}}$ se denominan cargas de los factores.

La cantidad apropiada de factores se extrajo por el método de Componentes Principales (CP), a partir de la matriz de correlación fenotípica de las características de tipo con valores propios mayores a 1 .

Para la interpretación de cada uno de los factores retenidos se tuvo en cuenta que los coeficientes de cada característica dentro del factor $\lambda_{\mathrm{jk}}$ fueran mayores a 0.30.

Se realizó una rotación de los factores por el método varimax propuesto por Kaiser (9), con el objetivo de lograr una interpretación adecuada de cada uno de los factores que se retuvieron.

Para evaluar la relación de los factores con la producción de leche, se utilizó un modelo lineal generalizado mediante el procedimiento GLM de SAS $\AA$ 9.1. El modelo incluyó los efectos fijos de hato, mes y año de parto y la regresión de cada uno de los factores retenidos por el procedimiento anterior. El modelo utilizado fue el siguiente:

$$
Y_{i j k m}=\mu+Y_{i}+c_{j}+\lambda_{k}+\beta_{2} f_{1 /}+\ldots . .+\beta_{n} f_{n l}+e_{i j k m}
$$

Donde y es la producción de leche ajustada a 305 días y edad madura, $\mu$ es la media de producción de leche, $y_{i}$ es el efecto fijo del i-ésimo hato, $\zeta_{j}$ es el efecto fijo de mes de parto, $\lambda_{k}$ es el efecto fijo de año de clasificación y $\beta_{2} f_{1 /}+\ldots+\beta_{n} f_{n /}$ hace referencia a los / factores retenidos de 1 a hasta $n y e_{i j k l m}$ es el error aleatorio.

\section{RESULTADOS}

Las medias y las desviaciones estándar para cada una de las 24 características de tipo evaluadas en la primera lactancia se pueden observar en la tabla 1 . Las medias variaron de 4.45 a 6.34 , donde los mayores coeficientes de variación se presentaron para las características ancho de la inserción (35.73) y vista posterior de los miembros (30.89)

Tabla 1. Medias, desviaciones estándar y coeficiente de variación de las características de tipo en vacas Holstein del departamento de Antioquia.

\begin{tabular}{lcc}
\hline \multicolumn{1}{c}{ Característica } & Media & CV \\
\hline Estatura & $5.98 \pm 1.27$ & 21.24 \\
Extremo anterior & $4.93 \pm 0.97$ & 19.68 \\
Tamaño & $5.63 \pm 1.31$ & 23.27 \\
Ancho de pecho & $4.96 \pm 1.36$ & 27.42 \\
Profundidad de cuerpo & $5.51 \pm 1.18$ & 21.42 \\
Fortaleza del lomo & $5.71 \pm 1.24$ & 21.72 \\
Colocación de ísquiones & $4.86 \pm 1.09$ & 22.43 \\
Ancho de ísquiones & $5.45 \pm 1.41$ & 25.87 \\
Ángulo de la pezuña & $4.82 \pm 1.25$ & 25.93 \\
Uniformidad de pezuña & $5.31 \pm 1.09$ & 20.53 \\
Profundidad de talón & $4.79 \pm 1.25$ & 26.10 \\
Calidad del hueso & $5.86 \pm 1.46$ & 24.91 \\
Colocación de los miembros & $5.71 \pm 1.25$ & 21.89 \\
Vista posterior & $5.18 \pm 1.60$ & 30.89 \\
Profundidad de la ubre & $5.48 \pm 0.98$ & 17.88 \\
Textura de la ubre & $6.22 \pm 1.25$ & 20.10 \\
Ligamento suspensorio medio & $6.31 \pm 1.25$ & 19.81 \\
Inserción anterior & $5.38 \pm 1.57$ & 29.18 \\
Colocación de pezones anteriores & $4.74 \pm 1.08$ & 22.78 \\
Largo del pezón & $4.89 \pm 1.20$ & 24.54 \\
Altura de la inserción posterior & $6.99 \pm 1.59$ & 35.73 \\
Ancho de la inserción & 17.15 \\
Colocación de pezones posteriores & 6.26 \\
\hline
\end{tabular}


Los análisis de factores realizados inicialmente mostraron que las características colocación de ísquiones, vista posterior de los miembros, largo del pezón, altura de la inserción posterior y fortaleza del lomo no tenían peso dentro de los factores con valores propios mayores que 1 , por lo que fueron excluidos del análisis y se realizó un nuevo análisis con las otras 19 características.

Se encontró una correlación aceptable y una adecuación muestral aceptable, según el coeficiente de KMO (Kaiser-MeyerOlkin), la cual fue de 0.75 .

Se encontraron siete valores propios mayores que 1 , los cuales explicaron el $64 \%$ de la variabilidad total de las 19 variables de tipo analizadas. El primer factor tuvo un valor propio de 3.85 y explicó el $20 \%$ de la varianza. El segundo y tercer factor obtuvieron valores propios de 2.05 y 1.70 con el $11 \%$ y $9 \%$, del total de la varianza, respectivamente.
La matriz de los factores rotados con aplicación de Varimax se puede observar en la tabla 2, donde valores superiores a 0.30 están marcados con un asterisco, indicando las características que definen ese factor.

El primer factor se relacionó con la estructura de la vaca, agrupando las vacas grandes, anchas de pecho y de ísquiones, altas, profundas del cuerpo, altas en su extremo anterior y angulosas. Las pezuñas, el sistema mamario, la vista posterior de la vaca, el carácter lechero, la ubre anterior y la profundidad de la ubre se relacionaron con los factores $2,3,4,5,6$, 7, respectivamente (Tabla 3).

En la tabla 4 se presentan los coeficientes de regresión lineal estándar de los factores que fueron retenidos y definidos en la tabla 3. El promedio de producción de leche fue de 7141.53 litros. Seis de los siete factores fueron altamente significativos con producción de leche, donde el factor

Tabla 2. Coeficientes de las características de tipo evaluadas en vacas Holstein del departamento de Antioquia en el Análisis de Factor.

\begin{tabular}{lccccccc}
\hline \multicolumn{1}{c}{ Característica } & Factor $\mathbf{1}$ & Factor $\mathbf{2}$ & Factor $\mathbf{3}$ & Factor $\mathbf{4}$ & Factor $\mathbf{5}$ & Factor $\mathbf{6}$ & Factor $\mathbf{7}$ \\
\hline Tamaño & $\mathbf{0 . 8 7}$ & 0.09 & 0.04 & 0.17 & -0.01 & 0.04 & -0.05 \\
Ancho de pecho & $\mathbf{0 . 7 5 *}$ & 0.06 & 0.02 & 0.10 & -0.26 & 0.08 & -0.15 \\
Profundidad del cuerpo & $\mathbf{0 . 6 3 *}$ & -0.16 & 0.05 & 0.14 & 0.11 & 0.19 & -0.29 \\
Estatura & $\mathbf{0 . 6 3 *}$ & 0.23 & 0.10 & 0.24 & 0.25 & -0.18 & 0.29 \\
Extremo anterior & $\mathbf{0 . 6 0 *}$ & -0.01 & -0.06 & -0.20 & 0.08 & $0.32 *$ & -0.04 \\
Ángulo de la pezuña & 0.03 & $\mathbf{0 . 8 6 *}$ & -0.01 & 0.05 & 0.01 & 0.05 & -0.05 \\
Profundidad del talón & 0.10 & $\mathbf{0 . 8 5 *}$ & 0.04 & 0.03 & -0.03 & 0.00 & 0.01 \\
Colocación de pezones posteriores & 0.04 & 0.03 & $\mathbf{0 . 8 2 *}$ & -0.19 & 0.01 & -0.03 & 0.01 \\
Ligamento suspensorio & 0.02 & -0.01 & $\mathbf{0 . 7 6 *}$ & 0.19 & 0.10 & 0.12 & -0.01 \\
Ancho de la inserción & 0.26 & -0.04 & 0.06 & $\mathbf{0 . 7 2 *}$ & 0.06 & 0.10 & -0.22 \\
Ancho de Ísquiones & $\mathbf{0 . 4 3 *}$ & 0.02 & 0.04 & $\mathbf{0 . 5 5 *}$ & 0.19 & -0.17 & 0.21 \\
Colocación de los miembros & 0.14 & -0.27 & 0.22 & $-\mathbf{0 . 5 2} *$ & 0.15 & -0.17 & 0.01 \\
Calidad de hueso & -0.19 & -0.07 & 0.02 & -0.02 & $\mathbf{0 . 8 4} *$ & 0.07 & 0.06 \\
Angularidad & $\mathbf{0 . 3 8 *}$ & 0.08 & 0.15 & 0.16 & $\mathbf{0 . 6 5} *$ & 0.14 & -0.17 \\
Inserción anterior & 0.16 & 0.04 & 0.01 & 0.00 & 0.09 & $\mathbf{0 . 8 2} *$ & 0.12 \\
Textura de la ubre & 0.05 & 0.16 & $\mathbf{0 . 3 2 *}$ & $\mathbf{0 . 3 5}$ & 0.23 & $\mathbf{0 . 5 1} *$ & -0.08 \\
Profundidad de la ubre & -0.14 & -0.06 & 0.01 & -0.09 & -0.03 & 0.14 & $\mathbf{0 . 8 7} *$ \\
Uniformidad de pezuñas & 0.09 & $\mathbf{0 . 3 4 *}$ & -0.03 & -0.06 & 0.08 & -0.04 & -0.22 \\
Colocación de pezones anteriores & 0.08 & -0.29 & $\mathbf{0 . 3 3 *}$ & 0.27 & -0.03 & $\mathbf{0 . 4 2 *}$ & 0.15 \\
\hline
\end{tabular}

*Valores absolutos mayores de 0.30 que explican cada uno de los factores. 
Tabla 3. Descripción de los factores obtenidos de las características de tipo evaluadas en vacas Holstein del departamento de Antioquia.

\begin{tabular}{|c|c|c|}
\hline Factor & Nombre & Característica \\
\hline 1 & Estructura de la vaca & $\begin{array}{l}\text { Vacas grandes, anchas de pecho y de ísquiones, } \\
\text { altas, profundas del cuerpo, altas en su extremo } \\
\text { anterior y angulosas. }\end{array}$ \\
\hline 2 & Pezuñas & $\begin{array}{l}\text { Vacas profundas de talón con pezuñas } \\
\text { empinadas y uniformes. }\end{array}$ \\
\hline 3 & Sistema mamario & $\begin{array}{l}\text { Vacas con pezones anteriores y posteriores } \\
\text { hacia adentro, ligamento suspensorio fuerte y } \\
\text { ubre con textura suave. }\end{array}$ \\
\hline 4 & Vista trasera de la vaca & $\begin{array}{l}\text { Vacas anchas en la inserción de la ubre, anchas } \\
\text { de ísquiones y patas derechas. }\end{array}$ \\
\hline 5 & Carácter lechero & Vacas angulosas y huesos planos \\
\hline 6 & Ubre anterior & $\begin{array}{l}\text { Vacas con inserción anterior de la ubre fuerte, } \\
\text { colocación de pezones anteriores hacia adentro } \\
\text { y textura de la ubre suave. }\end{array}$ \\
\hline 7 & Profundidad de la ubre & Vacas con ubres superficiales \\
\hline
\end{tabular}

Tabla 4. Coeficientes de regresión lineal para producción de leche ajustada a los 305 dias y edad adulta sobre los factores obtenidos de las características lineales evaluadas en vacas Holstein del departamento de Antioquia.

\begin{tabular}{cc}
\hline Factor & Coeficiente de regresión lineal \\
\hline F1 & $284.46 \pm 26.91^{* *}$ \\
F2 & $116.16 \pm 27.10^{* *}$ \\
F3 & $149.11 \pm 25.33^{* *}$ \\
F4 & $324.37 \pm 28.31^{* *}$ \\
F5 & $157.62 \pm 25.57^{* *}$ \\
F6 & $0.39 \pm 25.62 \mathrm{~ns}$ \\
F7 & $-356.17 \pm 25.39 * *$ \\
\hline
\end{tabular}

seis no fue significativo. El coeficiente de regresión del séptimo factor fue negativo, el resto de los coeficientes de regresión para los otros factores retenidos fueron positivos.

\section{DISCUSIÓN}

Los coeficientes de variación encontrados en este estudio fueron altos (entre 14.51 y 35.73), resultado cercano al reportado por Foster et al (10) quienes utilizando el sistema de medida estadounidense de 1 a 50 encontraron coeficientes de variación para las características de tipo entre 17.04 y 27.88 , lo cual indica que es normal encontrar en las características de tipo una heterogeneidad en sus valores debido al sistema de medición que se basa en medidas visuales realizadas por un clasificador.

Se presentaron siete factores con valores propios mayores a 1, los cuales explicaron un $64 \%$ de la variabilidad total. Este resultado concuerda con estudios similares realizados en características de tipo en bovinos Holstein de Estados Unidos, donde el total de la variabilidad obtenida en los factores retenidos explica entre el $69 \%$ y el $74 \%$ de la variabilidad total con menor número de variables $(11,12)$.

De los siete factores, el primer factor (estructura de la vaca) explicó el $20 \%$ de la variabilidad total, el cual estuvo conformado por vacas grandes y fuertes. Teniendo en cuenta el coeficiente de regresión lineal para este factor, se concluye que las vacas grandes y fuertes presentan una mayor producción de leche, resultado que concuerda con las correlaciones reportadas por Berry et al (2), quienes reportaron correlación genética positiva de la estatura, ancho de pecho, profundidad del cuerpo y ancho de ísquiones con producción de leche y difieren de lo encontrado por Sieber et al (11), quienes encontraron que vacas grandes y fuertes tenían una menor producción de leche. 
El segundo factor (conformación de pezuñas) explicó el $11 \%$ de la variabilidad total, el cual se caracterizó por vacas con pezuñas empinadas, uniformes y con un talón profundo. Estos resultados concuerdan con los encontrados por Sieber et al $(11,13)$ y Vukasinovic et al $(12)$, quienes reportaron que las características de las patas y pezuñas se agrupaban en un factor. Esto indica una fuerte correlación entre estas dos características. El análisis de varianza indicó una relación positiva (coeficiente de regresión positivo) de la producción de leche con vacas que tuvieron talón profundo, pezuñas angulosas y uniformes, to cual se explica por las condiciones de producción en Colombia, donde el sistema se basa principalmente en pastoreo en campos ondulados y las vacas con mejores condiciones de pezuñas son las que finalmente se desplazan más fácilmente desde el potrero hasta la sala de ordeño.

EL tercer factor (sistema mamario) se caracterizó por vacas con colocación de pezones hacia adentro, ligamento suspensorio fuerte y ubre con textura suave. El cuarto factor (vista trasera de la vaca) se definió por vacas anchas de la inserción de la ubre, de ísquiones y patas derechas; el quinto factor (carácter lechero) por vacas angulosas y de huesos planos. Los coeficientes de regresión positivos de los factores 3,4 y 5 indicaron que vacas con buena conformación de la ubre, tren posterior y con carácter lechero tienden a tener mayores producciones de leche.

El sexto factor (ubre anterior) en la cual las vacas se caracterizaron por tener una inserción fuerte, pezones hacia adentro y textura de la ubre suave, presentó un coeficiente de regresión con producción de leche negativo, pero no fue significativo.

El sexto factor (ubre anterior) en la cual las vacas se caracterizaron por tener una inserción fuerte, pezones hacia adentro y textura de la ubre suave, presentó un coeficiente de regresión no significativo con producción de leche. Esto se debe principalmente a que estas características no se relacionan con la capacidad de almacenar o producir leche y por el contrario se encuentran más relacionadas con la vida productiva de la vaca (12).

El séptimo factor (profundidad de la ubre) se caracterizó por vacas con ubres superficiales. Este factor obtuvo un coeficiente de regresión negativo con producción de leche, lo cual indicó que las vacas con ubres superficiales tienden a tener menor producción de leche. Estos resultados concuerdan con los presentados por Sieber et al (11), quienes encontraron que las vacas con ubres superficiales tenían un coeficiente de regresión negativo con producción. Lo anterior puede ser explicado por la poca capacidad que tiene la ubre de almacenar leche en gran cantidad debido a lo estrecha en la inserción y en lo superficial de la ubre. Además, la vaca al tener una profundidad del cuerpo muy superficial, no tiene la capacidad para almacenar grandes cantidades de alimento.

Finalmente, se puede concluir que los individuos con buenas condiciones corporales tienden a producir mayores cantidades de leche. Sin embargo, se hace necesario la realización de estudios tendientes a evaluar los puntajes ideales en condiciones locales y de esa manera poder relacionar los resultados encontrados con los reportados en otros países.

\section{Agradecimientos}

Este trabajo fue posible gracias al apoyo del Ministerio de Agricultura y Desarrollo Rural, Fondo Nacional del Ganado, Universidad de Antioquia, Asociación Holstein de Colombia y Corporación Antioquia Holstein. 


\section{REFERENCIAS}

1. Wiggans GR, Gengier N, Wright JR. Type trait (Co)Variance components for five dairy breeds. J Dairy Sci 2004; 87:2324-2330.

2. Berry DP, Buckley F, Dillon P, Evans RD, Veerkamp RF. Genetic relationships among linear type traits, milk yield, body weight, fertility and somatic cell count in primiparous dairy cows. Ir J Agr Food Res 2004; 43: 161-176.

3. Samoré $A B$, Rizzi R, Rossoni A, Bagnato A. Genetic parameters for funtional longevity, type traits, somatic cell scores, milk flow and production in the Italian Brown Swiss. Ital J Anim Sci 2010; 9:145-152

4. Daliri Z, Hafezian SH, Parvar A, Rahimi G. Genetic relationships among longevity, milk production and linear type traits in Iranian Holstein cattle. J Anim Vet Adv 2008; 7(4):512-515.

5. López E. Tratamiento de la colinealidad en regresión lineal múltiple. Psicothema 1998; 10: 491-507.

6. Macciota NP, Vicario D, Corrado DM, Caprio-Borlino A. A multivariate approach to modeling shapes of individual lactation curves in cattle. J Dairy Sci 2004; 87:1092-1098.

7. Thompson B. Exploratory and confirmatory factor analysis: Understanding concepts and applications. International Standard Book Number: 1-59147-093-5. Washington DC, USA: American Psychological Association; 2004.
8. Stanton $T L$, Blacke RW, Quaas RL, Van Vleck LD, Carbaño MJ. Genotype by environment interaction for Holstein milk yield in Colombia, Mexico, and Puerto Rico. J Dairy Sci 1991; 74:17001714.

9. Kaiser HF. The varimax criterion for analysis rotation in factor analysis. Psychometrika 1958; 23:187-200.

10. Foster $W W$, Freeman $A E$, Berger PJ. Linear type trait analysis with genetic parameter estimation. J Dairy Sci $1988 ; 71: 223-231$.

11. Sieber M, Freeman AE, Hinz PN. Factor analysis for evaluating relationships between first lactation type scores and production data of Holstein Dairy cows. J Dairy Sci 1987; 70:1018 - 1026.

12. Vukasinovic N, Moll J, Künzi N. Factor analysis for evaluating relationships between herd life and type traits in Swiss Brown cattle. Livest Prod Sci 1997; 49:227-234.

13. Sieber $M$, Freeman $A E$, Hinz PN. Comparison between factor analysis from a phenotypic and genetic correlation matrix using linear type traits of Holstein dairy cows. J Dairy Sci $1988 ; 71: 477-484$. 